\title{
Detection of Malignant Tissue using Metal Dielectric Interface Based Plasmonic Biosensor
}

\author{
Nandhini V L, Sandip Kumar Roy, K. Suresh Babu
}

\begin{abstract}
Metal-dielectric interface based Plasmonic biosensors commonly known as Surface Plasmonic Resonance (SPR) used in numerous applications in the analysis of bio reagents interaction and detection of the biological pathogen, cells and provides the advantage of real-time label-free efficient method. Sensitivity is a key parameter to measure sensor performance during development. Among all the plasmonic configurations, waveguide coupled configuration is most effective. In such a configuration an SPR and a waveguide are coupled through an evanescent field. This provides superior control over the biological reagent interaction, greater sensitivity and a multichannel-robust sensing device. Such devices are very small in size, lightweight as compared to prism coupled configuration which is bulky. These devices are also equipped to segregate specific sensor responses from non-specific response simultaneous multi analytes analysis. In view of the aforesaid benefits of waveguide coupled SPR, our present work focuses on a dual channel integrated optical waveguide based on metal dielectric interface biosensor. To study the behaviour of a waveguide coupled SPR sensor, we carried out analysis and simulation of SPR on multilayer geometries using tumourassociated antigens (TAAs). First, we started with the analysis of an SPR in the multi-layer intersection and observed the effect of variation of material characteristics on SPR profile. Further, extended our investigation to multilayered optical waveguide and the propagation constant was calculated. TAA and Newton's method were used for Lung Cancer and Breast Cancer infected tissue and sensitivity 300nm/RIU obtained.
\end{abstract}

Keywords:- Biosensor, Surface Plasmon Resonance, Refractive Index, Photonic Crystal, Malignant, Dielectric Interface, Plasmonic.

\section{INTRODUCTION}

Optical biosensors are extensively used in biomedical science, healthcare industry, and medicine companies. Optical biosensors are also implemented in environmental agencies and homeland security. The main reason to use optical biosensors is that optical sensors are immune to interference from electric and magnetic fields, capable of sensing remotely and can provide multiple detection capabilities in one chip.

Optical bio sensing involves two type of techniques fluorescence and label-free, in the former technique, bio molecules are labeled with dye tags and in the later one no bio marker are used. The optical sensor detects presence of the target molecule by the intensity of the fluorescence and the coupling with the bio recognition molecules. This technique is highly accurate (accuracy level of a single molecule), but involves laborious labeling processes. In addition, the labeling process may hinder with the normal functioning of the biomolecule leading to uncontrolled fluorophores. On the other hand, in case of label free technique, no labeling / no alteration of molecules is required. The label free method is gaining more attention because it provides quantitative molecular interaction evaluation with lesser cost and labor intensive implementation.

In the context of current work, Refractive Index (RI) change influenced by dynamics of molecular behavior alteration is monitored. The molecular behavior change may be outcome of not only sample's mass but also from variation of material properties. The output is independent of the scale of the sample volume. This characteristic is key for Nanoscale sensing devices. In the case of fluorescence based method, the output spectrum depends on the total number of analytes in the sensing area. Eventhough there are glaring differences between the two methods, both are extensively used to in sensing applications for molecular inter. Optical biosensing techniques are more diverse in nature than others techniques. The biosensing Lab-On-Chip is designed for a sensor that can detect cancer [1] affected area with a particular set of the tissues. Although Photonic Crystals based sensing technology is emerging, SPR biosensors [2] also gaining significant interest from researchers in the advancement of SPR technology, primarily because of more accurate results when compared to Photonic Crystals.

We have used two case studies one for Lung Cancer (Lung Carcinoma) and other for Breast Cancer. Lung carcinoma is result of uncontrolled cell growth in lung tissues. During the last decade the mortality rate because of Lung Carcinoma is ever increasing and immediate measure on early diagnosis (prognosis) may bring the down the mortality rate. Prior to clinical diagnosis if a sensor can detect the initial symptom of the tissue at microcellular level then only the prognosis will be useful[3]. For cancer-related mortality in women, breast cancer is one of the foremost reason. Eventhough, screening with mammography is widely used in clinical diagnosis, the prognosis still remains a challenge [4][5].

Revised Manuscript Received on April 12, 2019.

Nandhini V L, ECE Department, Govt. SKSJTI College, Bangalore, Karnataka, India. (sunandi7276@gmail.com)

Dr Sandip Kumar Roy, ECE Department, AMC Engineering College, Bangalore, Karnataka India. (sandipr@hotmail.com)

Dr K. Suresh Babu, ECE Department, UVCE College, Bangalore, Karnataka India. (ksb1559@gmail.com) 


\section{DETECTION OF MALIGNANT TISSUE USING METAL DIELECTRIC INTERFACE BASED PLASMONIC BIOSENSOR}

\section{WORKING PRINCIPLE}

The Fig.1 illustrates the basic block diagram of a Biosensor. The input of a biosensor contains a Gaussian pulse light source so that the transmission spectrum can be observed. The transducer in the block diagram transforms the sensing information with the change in RI of the thin film - the basic principle of SPR phenomenon [6]. Electromagnetic (EM) waves which are passed through the border between the two media that consists of the different dielectric constants result in the generation of the evanescent waves and the field penetrates into both the media which exponentially decays and is a function of distance from the intersection. It results in the creation of surface plasmon oscillations or waves when one of the regions is made up of dielectric material and other is made of metal.

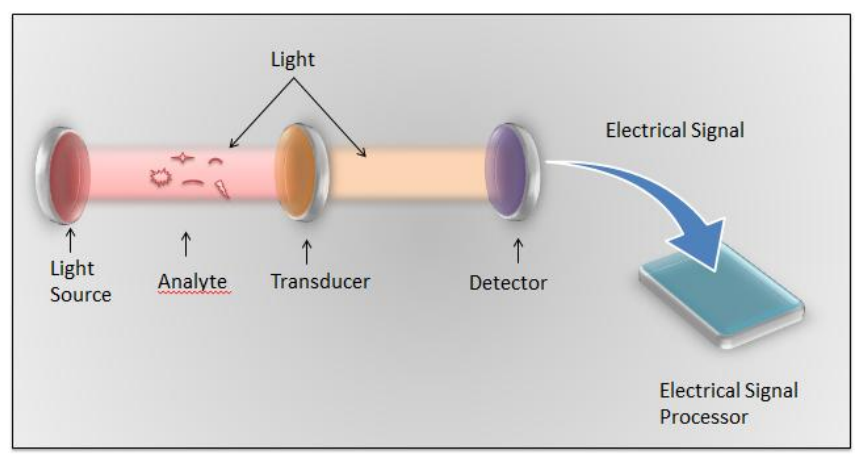

Fig 1: Basic Block Diagram of Biosensor

By using the reflected p-polarized light source the surface plasmons will be excited. When the incident source matches with that of the frequency of the metal layer there is an entanglement of light with the surface plasmons thereby causing the electronic resonance. The energy absorption will further reduce the intensity of the reflected light source this reduction takes place at a resonant wavelength. When the metal surface RI is combined with this there is a variation in the resonant wavelength. Thus, the biomolecular interaction can be observed in terms of wavelength change which takes place in the process of biological response. Sensors which are developed using SPR technology gives multichannel, highly integrated devices since the structure has the capability to involve many sensing components placed on one substrate [7]. The detection system (detector and signal processing integrated circuits) is employed in order to record the intensity of the reflected light at the output section of the waveguide and dip positions will be noted for the purpose of further analysis.

When a thin metalic film placed at the intersection between two materials of different RI, SPR gets initiated. In an SPR device, a 50-nm gold film is placed between the glass layers and the sample solution passed through the intersection above the metal film. As shown in Fig. 2, a near-infrared LED with focused plane polarized light is used as source and a diode array was placed to detect the intensity of the reflected light (total internal reflection). During SPR condition, the light penetrates an evanescent wave(EM component) across the metalic interface into the analyte. When the light is incident at a certain critical angle (SPR angle), the electrons of the conducting film gets excited resulting surface plasmons (electron charge density waves) formation within the metalic film. At SPR angle, reflected light intensity gets dropped. The SPR angle shifts when there is a variation in mass because of change in RI near the sensor surface.

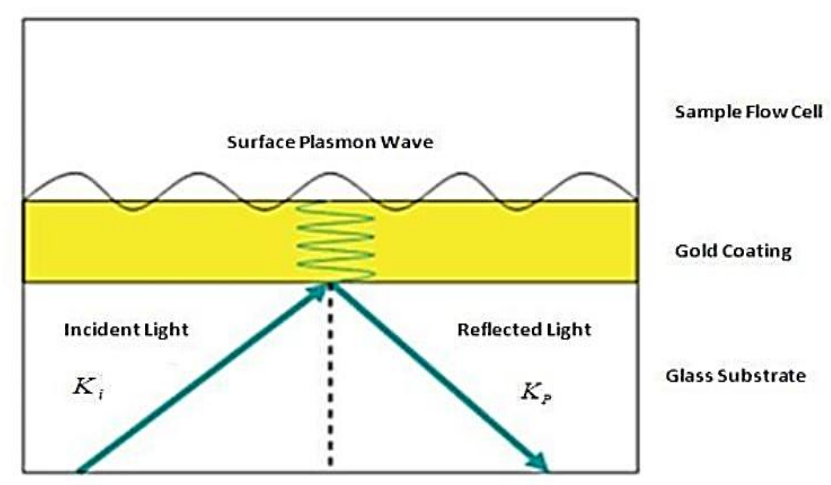

Fig 2 Surface Plasmon resonance

During SPR phenomenon, at the border of two media, there will be an oscillation of charges. At the border, the charge density field vector reaches its peak and then deteriorates into the media resulting a TM-polarized wave. By applying suitable EM boundary specifications at the interface, we can derive the Surface Plasmon propagation(SPP) constant and is as follows:

$$
\beta_{s p}=\frac{\omega}{c \sqrt{\left.\left\{\left(\varepsilon_{\text {metal }} * \varepsilon_{\text {dielectric }}\right) / \varepsilon_{\text {metal }}+\varepsilon_{\text {dielectric }}\right)\right\}}}
$$

Where,

$\omega$ - Angular frequency

c - Speed of light $=3 * 108 \mathrm{~m} / \mathrm{s}$

$\varepsilon_{\text {metal }}$ - Permittivity of metal

$\varepsilon_{\text {dielectric }}-$ Dielectric Permittivity

We have used gold as a metalic layer because Gold's permittivity is complex and vary based on wavelength.

\section{RESULTS \& DISCUSSIONS}

In order to design the metal-dielectric intersection based plasmonic biosensor to detect malignant tissues, Opti-FDTD and R-Soft design tools were used.

Opti-FDTD is a robust, highly programmed and userfriendly software program that empowers the computeraided design (CAD) and simulation of progressive passive and nonlinear photonic components. Opti-FDTD helps to model, analyze, design, develop and test contemporary passive and nonlinear photonic components for wave propagation, scattering, diffraction, polarization and the nonlinear phenomena. The core program of Opti- FDTD is a Finite Difference Time Domain (FDTD) algorithm with second-order numerical accuracy and most advanced border conditions. Opti-FDTD empowers the simulation of Photonic Band Gap (PBG) of materials, optical micro ring filters, ring resonators in multiple structures, waveguides and other nano scale photonic elements. 
The R-Soft CAD is part of the R-Soft Photonics Suite and is a control program for R-Soft's modules for passive device simulation. The FullWAVE software uses the FDTD method for simulation. This helps to do an analysis of devices, such as PBG and ring resonators [8]. R-Soft implements highly accurate algorithms, assists in "what if" product scheme and scripting can be done with any programming language at the available common CAD interface.

Flowchart of the R-Soft Software based sensor design and simulation is shown in Figure 3. It is required to define the material, initial simulation domain properties and waveguide profile. The aim is to design system parameters such a way that on the incident of Gaussian Pulse on the metal-dielectric intersection, the plasmonic oscillation happens.

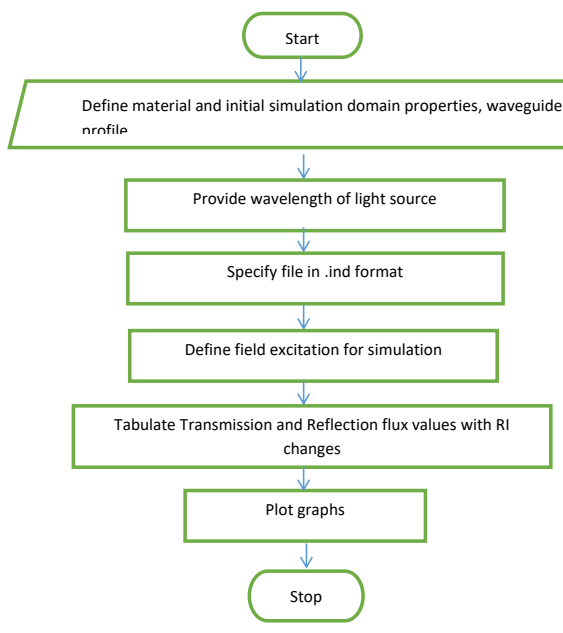

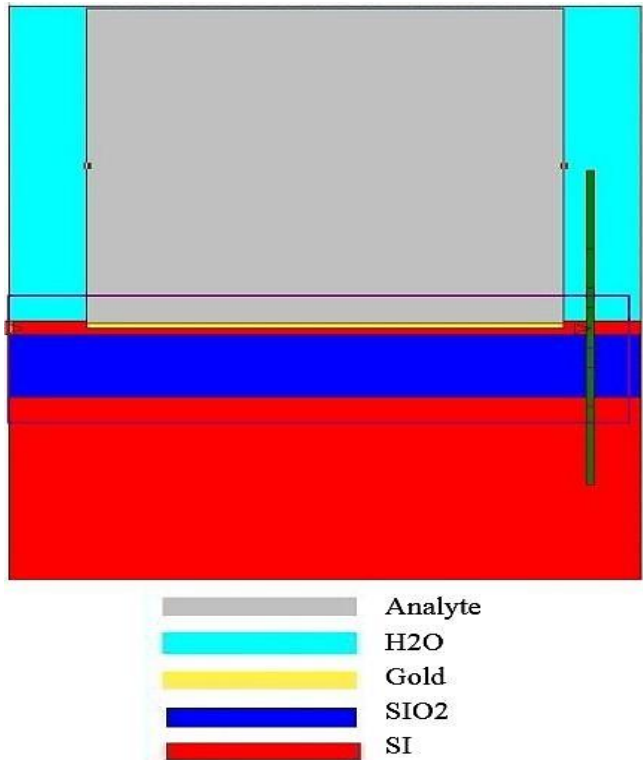

(a)

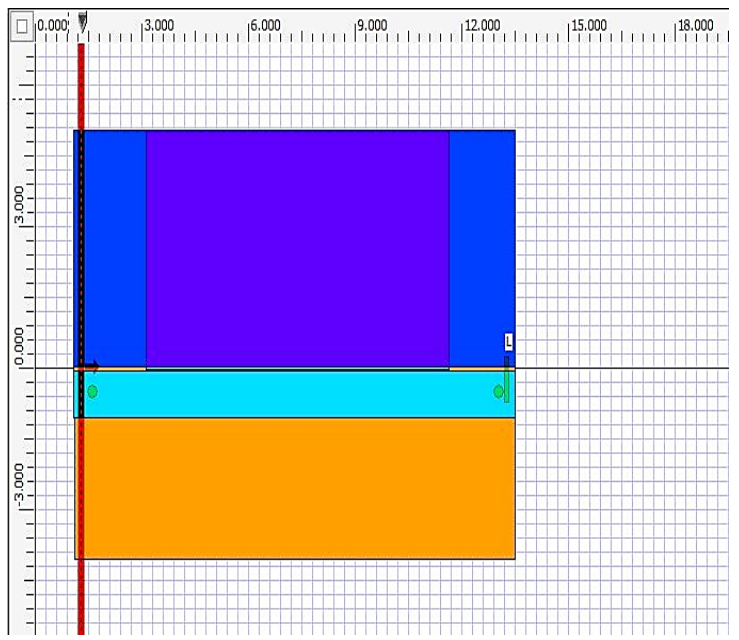

(b)

\section{Fig 3: Flowchart of the Software}

The figure below illustrates the design of multi-layer structure sensor design using R-Soft tool and also OptiFDTD. Figure 4 shows the designed waveguide for the SPR biosensor. As and when the light is incident through an optical source on the gold coating, the surface plasmonic resonance takes place. This phenomenon occurs due to the interaction of resonant oscillation of conduction electrons stimulated by incident light at the at the interface between material layers with -ve and +ve permittivity. When the surface plasmon wave(SPW) interacts with an analyte, part of their energy re-emitted as light. This emitted light through the metal film provides information about molecular binding and used for sensing the property of the sample analyte.

Fig 4: Design of Multi-Layer structure Sensor Design using (a) R-Soft (b) Opti-FDTD

The simulation is shown in Fig.5 below. One of the noble metal considered here is the gold coating. Due to the presence of localized surface plasmon oscillations, nanoparticles within the sensing medium, intense colour spots are there. Nanoparticles of gold shows strong absorption bands in the ultraviolet to visible light spectrum. Shifts in this resonance due to changes in the $\mathrm{RI}$ is the principle behind detection of malignant tissue using the metal-dielectric interface biosensor. Table 1 provides the design specification. 
TABLE 1: Design specification

\begin{tabular}{|l|c|c|}
\hline $\begin{array}{l}\text { Waveguide } \\
\text { Layer }\end{array}$ & $\begin{array}{c}\text { Refractive } \\
\text { Index(1.55} \boldsymbol{\mu m})\end{array}$ & Thickness \\
\hline $\mathbf{S i O}_{\mathbf{2}}$ & 1.45 & 2 \\
\hline Lower $\mathbf{S i}$ & 3.45 & 0.22 \\
\hline Upper Si & 3.45 & 0.101 \\
\hline Gold(Au) & 0.574 & 0.074 \\
\hline
\end{tabular}

Contour Map of Hy
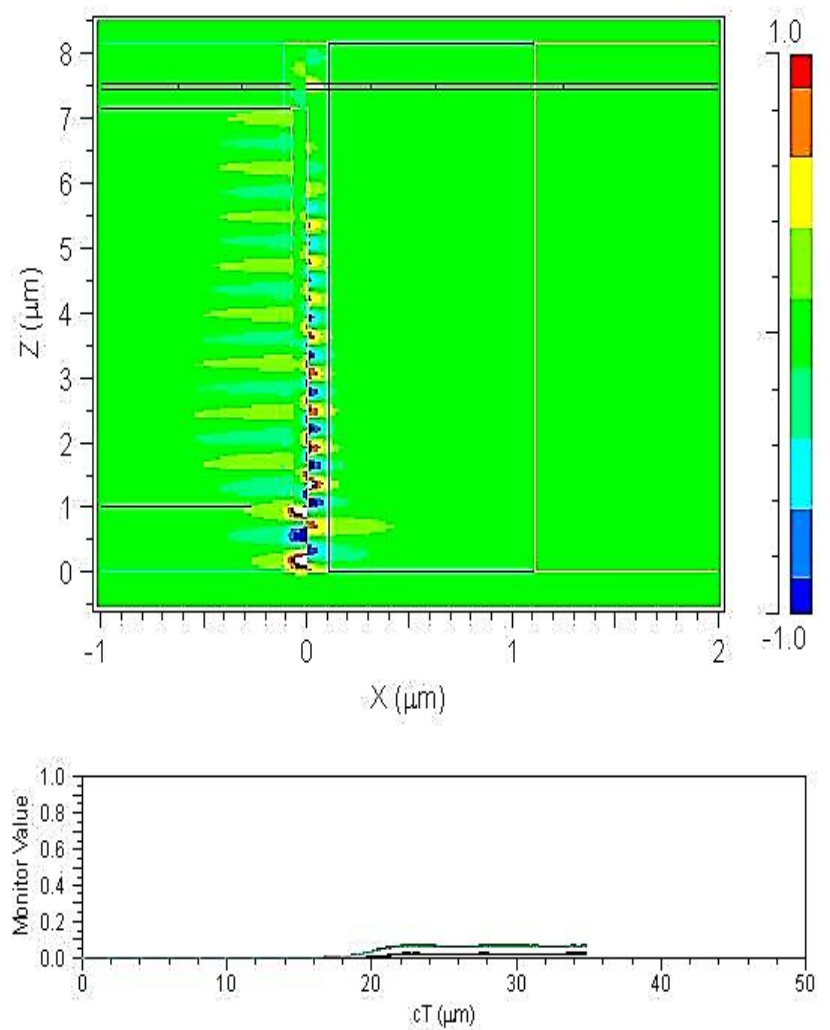

Fig 5: Snapshot of Simulation Window

Nano cavity biosensor for different cancerous tissue is analyzed in different metal layers. The software's used are R-Soft and Opti-FDTD. The same design is being simulated using two different software's to analyses various factors such as sensitivity, frequency analysis, transmission spectrum analysis and power spectrum analysis.

The sensor designed using the SPR technology consists of 4- layers (Gold, Silicon, Silicon Dioxide and Silicon as the substrate). Here the substrate layer is used to provide mechanical strength to the sensor. These waveguides with metals and have the capability to absorb the light and the segments are placed parallel to each other. The gold layer is placed above the silicon layer and it is excited by the input light source. When light interacts with this sensing arm we can observe the shift in the input light when detected by the detector

\section{SIMULATION}

Simulation of current work involves MEEP (Maxwell's Electromagnetic Equation Propagation) software package(open source) for EM simulation via the FDTD method spanning a broad range of applications. MEEP uses common standard languages and FDTD implementation can be done in $1 \mathrm{D}, 2 \mathrm{D} \& 3 \mathrm{D}$. Field analysis (Poynting flux, mode decomposition, energy density), sensitivity analysis and automated design optimization can be done using MEEP [9].

The objective of current work was to develop a sensor that can be miniaturized and integrated is necessary which can be configured to operate in multi-analyte mode. The SPR Sensor is the gold dielectric interface with $\mathrm{Au}$ film delivers steady sensing capability and excellent resistance to chemicals.

We have created a dual channel-based sensor using high RI material such as $\mathrm{Si}$ so that the device can be miniaturized and integrated for sensing application. We have used two case studies one for Lung Cancer and other for Breast cancer. Fig. 6 to Fig. 7 shows the resultant output of the sensing action. To analyze the intensity levels of transmission and reflection spectrums we have used MEEP and MATLAB. It has been observed that for small RI change, a significant frequency shift occurred leading to the conclusion that the developed device can be used as a sensor and can differentiate between different components of the normal and cancerous cell.
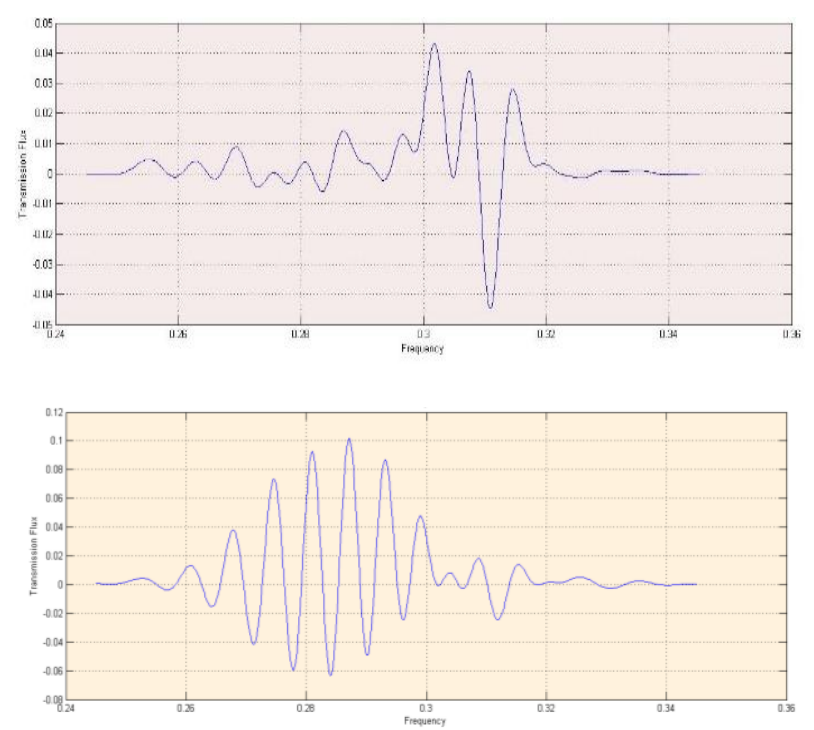

Figure 6 Spectrum analysis for normal(a) and malignant lung cancer (b)

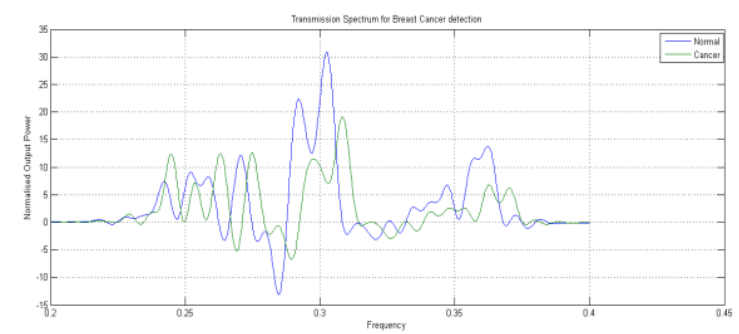

Figure 7 Spectrum analysis for Normal and Breast cancer

In the above graph, we can observe that as we change the test analyte from normal cell to cancerous cell there is wavelength shift indicating that the sensor is detecting the Melanoma cell. Here the sensitivity obtained is $300 \mathrm{~nm} / \mathrm{RIU}$. 


\section{CONCLUSION}

Design of metal-dielectric intersection based SPR sensor to detect malignant tissue is presented in this paper. The most commonly used gold-dielectric intersection based SPR sensor is used because the tiny Au metal layer provides excellent performance and high degree of chemical resistance. The sensor is miniaturized so that the same can be integrated into Nanoscale Lab-onChip and configured to operate in multi-analyte mode. Sensor structure design was done using R-Soft and OptiFDTD and simulation was done using MEEP for Cancer infected cell diagnosis. The objective was to do prognosis lung and breast cancer infected tissue. Because of the variation in RI between cancerous and non-cancerous cells, a distinct frequency shift is monitored in the transmission spectrum of normal and cancer infected tissues. From the presented result, we can draw inference that the preseted sensor design could detect cancer. Here the sensitivity obtained is $300 \mathrm{~nm} / \mathrm{RIU}$.

The outcome of current research can be used for Nano scale, low cost and less power hugry Lab-On-A-Chip design fabrication.

\section{REFERENCES}

1. H Patil, R Nischitha, TS Indumathi, P Sharan, "Liquid sensor based bio-chip for DNA analysis of cancer using photonic crystal" International Conference on Optical and Photonic Engineering (icOPEN2015).

2. S. K. Roy, Nethravathi, P. Sharan "Surface Plasmon Resonance (SPR) Based Dual Channel Optical Waveguide for Bio sensing Application", Recent Trends in Sensor Research and Technology. 2016; 3(2): 1-7p.

3. W. Lai et al., "Silicon photonic crystal microcavity biosensors for label free highly sensitive and specific lung cancer detection," IEEE Photonics Conference 2012, Burlingame, CA, 2012, pp. 443-444.

4. S. Campuzano, M. Pedrero and J. M. Pingarrón, "NonInvasive Breast Cancer Diagnosis through Electrochemical Biosensing at Different Molecular Levels", Sensors 2017, 17(9), 1993; doi: 10.3390/s17091993.

5. S. Roy, P. Sharan, "Photonic Crystal Based Sensor for DNA Analysis of Cancer Detection". In: Mishra A., Basu A., Tyagi V. (eds) Silicon Photonics \& High-Performance Computing. Advances in Intelligent Systems and Computing, vol 718. 79-85, 2018.

6. J. Homola, "Optical-fiber sensor-based on surface-plasmon excitation", Sensors and Actuators B: Chemical, Volume 29, Issues 1-3, October 1995, Pages 401-405.

7. B. D. Terris, H. J. Mamin, W. R. Studenmund, and G. S. Kino, "Near-field optical data storage using a solid immersion lens", Appl. Phys. Lett. 65(4), 388-390 (1994).

8. A. Raganna, S.H. Bharathi, S. Roy, P. Sharan, "Rapid Detection of Drugs Abuse by Adapting Surface Plasmonic Micro Ring Resonator". International Journal of Signal Processing, Image Processing and Pattern Recognition. 10. 111-124. 10.14257/ijsip.2017.10.8.10. 2017.

9. P. Sharma, P. Sharan, "An optical sensor for propagation analysis of Lymphocyte cell for cancer cell detection". 2014 IEEE Global Humanitarian Technology Conference South Asia Satellite, GHTC-SAS 2014. 93-98. 10.1109/GHTC-SAS.2014.6967565. 2014

10. E. X. Jin, and X. Xu, "Finitte-Difference Time-Domain Studies on Optical Transmission through Planar NanoApertures in a Metal Film,” Jpn. J. Appl. Phys. 43(1), 407417 (2004)
11. F. D. Gudagunti, P. Sharan, S. Talabattula and Nainitej V, "Early stage detection of breast cancer using hybrid photonic crystal ring resonator," 2014 IEEE International Conference on Advanced Communications, Control and Computing Technologies, Ramanathapuram, 2014, pp. 1542-1545. doi: 10.1109/ICACCCT.2014.7019364.

12. S. Chaitra, C. Veena, K. Rao, P. Sharan, "SPR based biosensor for the detection of abnormal growth of tissues". 149-153. 10.1109/ICNETS2.2017.8067918. 2017.

13. P. Sharma and P. Sharan, "An optical sensor for propagation analysis of Lymphocyte cell for cancer cell detection," 2014 IEEE Global Humanitarian Technology Conference - South Asia Satellite (GHTC-SAS), Trivandrum, 2014, pp. 93-98, 2014.

14. Savitha, K. S. Rao and P. Sharan, "Detection of oncological cell for breast cancer by using SPR technology," 2016 3rd International Conference on Computing for Sustainable Global Development (INDIACom), New Delhi, pp. 2747-2749. 2016.

15. Nithin S, P. Sharma, Vivek M and P. Sharan, "Automated cervical cancer detection using photonic crystal based biosensor," 2015 IEEE International Advance Computing Conference (IACC), Bangalore, pp. 1174-1178. doi: 10.1109/IADCC.2015.7154888, 2015.

16. R. C. Jorgenson, and S. S. Yee, "A fibre optic chemical sensor based on surface Plasmon resonance", Sensors and Actuators B: Chemical, Volume 12, Issue 3, 15 April 1993, Pages 213-220.

17. H. H. Nguyen, J. Park, S. Kang and M. Kim "Surface Plasmon Resonance: A Versatile Technique for Biosensor Applications", Sensors, 15, 10481-10510; doi: 10.3390/s 150510481, 2015

18. Y. Yanase, T. Hiragun, K. Ishii, T. Kawaguchi, T. Yanase, M. Kawai, K. Sakamoto and M. Hide "Surface Plasmon Resonance for Cell-Based Clinical Diagnosis", Sensors, 14, 4948-4959; doi:10.3390/s140304948, 2014.

19. K. Q. Bienstman, Enhanced Sensitivity of Silicon-OnInsulator Surface Plasmon Interferometer with Additional Silicon Layer. IEEE Photonics Journal, Vol. 3, No. 3, 538 $545,(2011)$

20. E. Petryayeva, U. J., 'Localized surface plasmon resonance: Nanostructures, bioassays and biosensing-A review', Analytica Chimica Acta, 8-24, (2011).

21. Geum-Yoon Oh, 1. T.-K.-S.-W. 'Design of ultra-sensitive biosensor applying surface plasmon resonance to a triangular resonator'. Optics express, 19067-19074, (13 August 2012).

22. J. Dostalek, J., 'Surface plasmon resonance biosensor based on integrated optical waveguide'. Sensors and Actuators B, 8-12, (2001).

23. Kun-Huang Chen, C.-C. H.-C., 'Measurement of wavelength shift by using surface plasmon resonance heterodyne interferometry. Optics Communications', 167172, (2002)

24. T. H. Yuhki Yanase, 'Surface Plasmon Resonance for CellBased Clinical Diagnosis. Sensors, 4948-4959, (2014).

25. D.R. Shankaran,; Gobi, K.V.; N. Miura, 'Recent advancements in surface plasmon resonance immunosensors for detection of small molecules of biomedical, food and environmental interest'. Sensors and Actuator. B, 121, 158-177, (2007). 